\title{
Intraventricular Hemorrhage with Parenchymal Hemorrhage of the Newborn
}

National Cancer Institute

\section{Source}

National Cancer Institute. Intraventricular Hemorrhage with Parenchymal Hemorrhage

of the Newborn. NCI Thesaurus. Code C117202.

Bleeding into the lateral cerebral ventricles and the adjacent brain tissue of a newborn infant. 